\title{
A droplet-based microfluidic platform for rapid immobilization of quantum dots on individual magnetic microbeads
}

\author{
Thu H. Nguyen ${ }^{1}$, Xiaoming Chen ${ }^{1}$, Abootaleb Sedighi ${ }^{2}$, Ulrich J. Krull ${ }^{2}$, Carolyn L. Ren ${ }^{1 *}$ \\ ${ }^{1}$ Department of Mechanical and Mechatronics Engineering, University of Waterloo, 200 \\ University Avenue West, Waterloo, Ontario, Canada \\ ${ }^{2}$ Department of Chemical and Physical Sciences, University of Toronto Mississauga, 3359 \\ Mississauga Road, Mississauga, Ontario, Canada
}

* Corresponding author:

Tel: $1-519-888-4567 \times 33030$

Fax: 1-519-888-4567

E-mail: c3ren@uwaterloo.ca

\begin{abstract}
Quantum dots (QDs) provide opportunities for development of bioassays, biosensors, and drug delivery strategies. Decoration of the surface of QDs offers unique functions such as resistance to non-specific adsorption, selective binding to target molecules and cellular uptake. The quality of decoration has substantial impact on the functionality of modified QDs. Single-phase microfluidic devices have been demonstrated for decorating QDs with biological molecules. The device substrate can serve as a solid phase reaction platform, with a limitation being difficulty in the realization of reproducible decoration at high density of coverage of QDs. Magnetic beads (MBs) have been explored as an alternative form of solid phase reaction platform for decorating QDs. As one example, controlled decoration to achieve unusually high density can be realized by first coating MBs with QDs, followed by addition of molecules such as DNA oligonucleotides. Uniformity and high density of coatings on QDs have been obtained using MBs for solid phase reactions in bulk solution, with the further advantage that the MBs offer simplification of procedural steps such as purification. This study explores the use of a droplet microfluidic platform to achieve solid phase decoration of MBs with QDs, offering control of local reaction conditions beyond that available in bulk solution reactions. A microchannel network with a two-junction in-series configuration was designed and optimized to co-encapsulate one single $1 \mu \mathrm{m}$ MB and many QDs into individual droplets. The microdroplet became the reaction vessel, and enhanced conjugation through the confined environment and fast mixing. A high density of QDs was coated onto the surface of single MB even when using a low concentration of QDs. This approach quickly produced decorated MBs, and significantly reduced QD waste, ameliorating the need to remove excess QDs. The methodology offers a degree of precision to control conjugation processes that cannot be attained in bulk synthesis methods. The proposed droplet microfluidic design can be widely adopted for nanomaterial synthesis using solid phase assays.
\end{abstract}

Keywords: Droplet microfluidics, quantum dots, electrostatic association, magnetic beads 


\section{Introduction}

In the last two decades, a promising nanomaterial, known as a semiconductor nanoparticle or quantum dot (QD), has demonstrated tremendous potential for enhancing imaging. Examples include applications such as fixed cell labeling, imaging of live cell dynamics, sensing and in vivo animal imaging (Bruchez Jr. et al. 1998; Zhang et al. 2005; Xing and Rao 2008; Algar et al. 2010; Sun and Gang 2013; Chou et al. 2014; He et al. 2014; Cao et al. 2016). QDs are chemically synthesized semiconductor nanocrystals with diameters of a few nanometers (2 to $10 \mathrm{~nm}$ ) and quantum yields that routinely approach $50-80 \%$. They are 100 times more resistant to photobleaching than molecular fluorescent dyes (Resch-Genger et al. 2008).

To endow QDs with selective binding capability, different biomolecules such as nucleic acids and antibodies that act as targeting agents or probes have been used to modify the surface of QDs via bioconjugation (Mattoussi et al. 2000; Zhang et al. 2005; Algar and Krull 2007; Resch-Genger et al. 2008). Such functionalized quantum dots have drawn a great deal of attention from both academia and industry because they enable the development of assays, in vitro diagnostics, and biosensors (Huo et al. 2006; Kim et al. 2009; Prabhu and Hudson 2009; Chou et al. 2014; Inoue et al. 2016; Liu et al. 2017). For instance, QDs functionalized with DNA oligonucleotides have been successfully used for biological applications within in vitro and in vivo environments (Boeneman et al. 2010; Lalander et al. 2010; Giri et al. 2011; Cutler et al. 2012; Noor et al. 2013a; Petryayeva et al. 2013; Stanisavljevic et al. 2015). QD-based DNA hybridization assays can operate on the basis of fluorescence resonance energy transfer (FRET) which often involves the conjugation of single-stranded DNA oligonucleotides (ssDNA) with QDs. A FRET pair can form where QDs are donors, and hybridization of the probe oligonucleotide with a target DNA strand that carries a fluorescent label serves as the acceptor (Zhang et al. 2005; Peng et al. 2007; Suzuki et al. 2008; Coopersmith et al. 2015). The sensitivity, selectivity and speed of binding of the ssDNA-QD conjugates depends on the density and structural uniformity of the oligonucleotide strands at the surface of the QDs.

One method to increase the deposition density is to first immobilize QDs onto the surface of magnetic beads (MBs) via electrostatic reaction, and then continuously load oligonucleotides onto the surface of QDs (Sedighi and Krull 2016). This solid phase conjugation strategy also offers the opportunity to concurrently decorate the QDs with different molecules meaning that the exposed part of the QD surface is coated with a particular probe ssDNA, and on release from the $\mathrm{MB}$, the other face of the QD can be coated with a different probe, or even a different class of molecule than an oligonucleotide. This strategy was implemented using a batch setting by bulk solution reaction. While functional, the batch operation imposes limitations. The batch approach does not allow multiple coating processes to be completed continuously. The reagent concentration is high to push reactions forward, but the consumption in each batch is small, resulting in high cost and large waste. The entire process is prone to variability due to changing conditions such as temperature, and to contamination (e.g. dust). Finally, yet importantly, purification is required to remove excess reagents and QD aggregates at each functionalization step in batch settings (Coopersmith et al. 2015). Microfluidics has potential to address these challenges by offering continuous processing using a confined environment for enhancing reactions, reducing reagent consumption, offering sample management for purification and efficient NP collection.

Single phase microfluidics that uses miscible fluids to transport samples and perform reactions has been reported for decoration of QDs, where the channel surface serves as the solid substrate for immobilizing QDs (Noor et al. 2013b). This work demonstrated that microfluidic-based solid phase reactions allowed nucleic acid hybridization within seconds to minutes due to small sample volumes, in contrast to bulk solution reactions where the identical hybridization reaction took hours to reach equilibrium. Despite its success, single phase microfluidics has some inherent limitations such as slow mixing, low throughput and high risk of cross contamination, which have largely limited the quantity and quality of the functionalized QDs and the opportunity to selectively decorate the surfaces of QDs. Droplet microfluidic methods that use an immiscible fluid to disperse the sample reagents into pico- to nanoliter droplets is capable of addressing these inherent limitations (Günther and Jensen 2006; Teh et al. 2008; Zhu and Fang 2013; Rosenfeld et al. 2014; Kaminski et al. 2016) while maintaining the continuous flow advantage. First, benefiting from the confinement of microchannels, monodispersed droplets can be generated at $\mathrm{kHz}$ rates offering the potential to handle a reasonable quantity of decorated QDs even when the volume per droplet is quite small. Second, three-dimensional (3D) motion occurs within the droplets, which provides almost instantaneous mixing enabling faster and more homogeneous reaction. Finally, selective decoration of the surface of QDs is possible by merging, splitting, trapping and releasing droplets in a controllable manner. Droplet microfluidics allows the integration of multiple reactions, as possible for the immobilization of QDs on solid substrates such as MBs, conjugation of 
biomolecules with the solution-facing surface of QDs, releasing the partially-decorated QDs from MBs, and conjugating other biomolecules onto the freshly exposed surface of QDs.

Even though the opinion of using the droplet-based microfluidics and the magnetic bead loading method to assist the nanoparticle decoration was mentioned in the review paper (Han et al. 2017), our reported work firstly advances the first step of a process to selectively decorate the surface of QDs by demonstrating the feasibility of using a droplet microfluidics platform to enhance rapid, high-density immobilization of QDs onto single $1 \mu \mathrm{m}$ MBs that serve as solid substrates. This step is critical to the subsequent processes and quality of bioconjugation, and thus the performance of QDs for biosensing and imaging. The ability to manipulate one single MB that is coated with QDs offers a degree of control of the conjugation conditions that is unattainable in batch settings. One question that arises is whether a droplet microfluidics approach is promising in ameliorating the need for QDs to reach uniform coating density on MBs. Therefore, effort has been made to characterize the quality of deposition by physical inspection using fluorescence microscopy.

\section{Working principle and Design of a microfluidic platform}

\subsection{Working principle}

To ensure a coating of QDs onto a MB, co-encapsulation of many QDs with only one single MB in one droplet must be achieved which is one of the goals of the design of the microfluidic system. The conjugation between QDs and MBs is via electrostatic attraction, which tends to be rapid and can be facilitated by enhancing mixing in the reaction environment. It is expected that the conjugation occurs within seconds, benefiting from the fast mixing within the droplet due to 3D motion. Electrostatic conjugation requires QDs and MBs to have opposite charges. This is realized by coating QDs with glutathione (GSH) to render surfaces negatively charged, and functionalizing the MBs with positively charged diethyllaminoethyl (DEAE) groups. The association of a magnetic bead with many QDs is referred to as the MB-QD conjugate. This process is schematically illustrated in Figure 1.

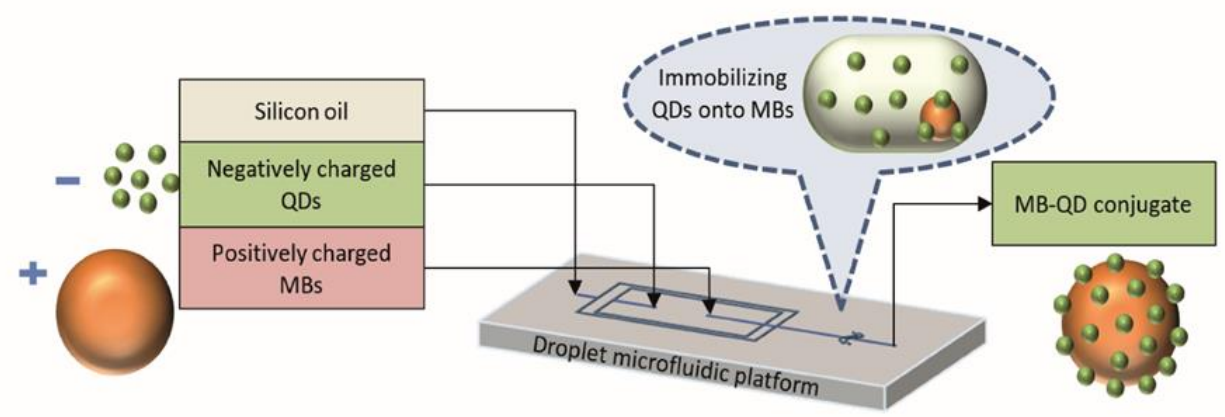

Fig 1. Schematic illustration of on-chip, in-droplet Magnetic Bead-Quantum Dots (MB-QD) conjugation via electrostatic adsorption

Monodispersed droplets can be generated using either passive or active methods (Teh et al. 2008; Chong et al. 2016; Zhu and Wang 2017), with each method offering opportunities and disadvantages. A passive method is chosen in this study to form monodispersed water-in-oil droplets because the design requires multiple pressure controls, which is simplified by operating with a multi-output pressure system (MFSC 4C, Fluent). In order to co-encapsulate one single magnetic bead and QDs, two junctions in series are designed. Two streams of aqueous buffer solutions with one carrying MBs and the other transporting QDs meet at the first junction forming a stratified flow, which is the dispersed phase. The continuous oil phase then interacts with the dispersed phase at the second junction forming monodispersed water-in-oil droplets. Details of this design are introduced later. 
Encapsulation of single micron-sized MBs is challenging. In general, each bead should be physically directed and sequentially ordered by using a focused flow. Some strategies have explored the use of gel materials to first encapsulate the beads (Tan and Takeuchi 2007; Um et al. 2008; Velasco et al. 2012) while others have used stratified flow with a viscosity contrast between the two fluids to focus the bead stream (Chen and Ren 2017). In the stratified flow design used herein, the inner fluid has a higher viscosity than the outer focusing fluid. At the beginning, the inner fluid moves more slowly than the outer fluid, resulting in a shear force at the interface. The shear force will speed up the flow of the inner phase until the velocity difference at the interface disappears. Under constant flow rates, the cross-section area of the inner fluid will decrease when the velocity increases, leading to a sharpened thin line of the inner phase ideal for focusing microbeads. In addition, the high viscosity inner fluid acts like a soft wall, which largely reduces the vortex inside the inner fluid, and therefore, prevents microbeads from moving out of the droplet during a droplet formation cycle.

The QD solution is used to focus the fluid carrying MBs at the first junction, resulting in a thin stream that allows one single MB of $1 \mu \mathrm{m}$ diameter to enter a solution that contains many QDs. These two solutions form the dispersed phase which is then formed into droplets, squeezed by the continuous oil phase at the second junction. Tuning of the flow and geometric parameters allows encapsulation of single MBs into individual droplets as illustrated in Figure 2.

\subsection{Design a microfluidic platform}

Initial work explored the in-series two-junction design for manipulation of relatively large polystyrene (PS) beads that had nominal diameters of $10 \mu \mathrm{m}$ and $4 \mu \mathrm{m}$. The design was demonstrated to operate successfully (see supplemental materials). Challenges arose when applying the same design and operational parameters for selecting individual $1 \mu \mathrm{m}$ MBs. The MBs were more sensitive to disturbance of flow than the larger PS beads, and refinement of the design and precise control of all the parameters affecting flow was required. The key was to ensure that the focused bead stream between the two junctions was not disturbed by any event occurring downstream, such as droplet formation in the channel network, which caused changes in local and global flow resistance. Attention has also been paid to ensure homogenous mixing. Several parameters that are widely applicable to single particle analysis influence this process.

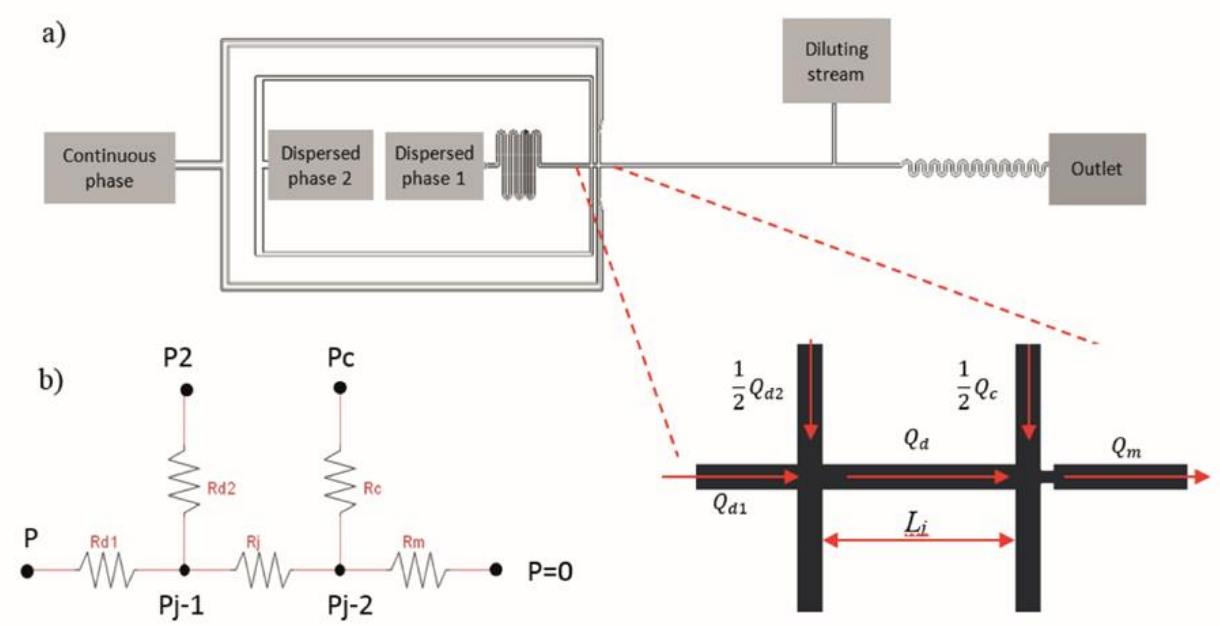

Fig 2. a) Layout of the droplet microfluidic platform for co-encapsulating a single MB and many QDs in individual droplets, with rapid mixing to enhance MB-QD conjugation, and b) The equivalent electrical circuit used to modify the microfluidic network.

The distance between the two junctions, $L_{j}$, is critical and should satisfy two main requirements: (1) allowing the stratified flow to fully develop, and (2) minimizing diffusion at the interface between the two miscible fluids. In addition, because the two junctions are coupled together, the hydrodynamic focusing in the first junction is also affected by the pressure change during the droplet formation cycle at the second junction (Garstecki et al. 2006; Chen et al. 2015). A design with the above concerns addressed is proposed using an electrical circuit analog (Oh et al. 2012) (Figure 2b). The microfluidic network is treated as a 1D circuit where the pressures applied at the inlets and 
outlets are considered as applied voltages, hydrodynamic resistances act like electrical circuit resistors and volumetric flow rate in each stream is treated as the electronic current. Kirchhoff's current law (KCL) and Kirchhoff's voltage law (KVL) are applied to analyze the circuit, and thus the operating parameters for the microfluidic network. In order to minimize the coupling effects caused by the pressure drop between the two junctions and maintain the hydrodynamic focusing of the bead stream, the length between the two junctions $\left(L_{j}\right)$ in our design is set at $300 \mu \mathrm{m}$. Smaller channel dimensions provide for improved spatial control of smaller particles such as $1 \mu \mathrm{m}$ MBs. However, the overall hydrodynamic resistance would increase dramatically, requiring much higher applied pressures to control the flow. In addition, smaller channels are prone to blockages. To balance these concerns, the channel height is set to be $25 \mu \mathrm{m}$, the width of the channels upstream and downstream are set to be $40 \mu \mathrm{m}$ and $50 \mu \mathrm{m}$, respectively. The microfluidic chip is made of polydimethylsiloxane (PDMS), which absorbs silicon oil. In practice, this results in slight swelling of the polymer and provides smaller channel heights than stipulated (i.e. $<25 \mu \mathrm{m}$ ), which has been considered in the design. In our study, the channels are primed with silicon oil for 20 minutes to reach saturation resulting in a channel height of $\sim 16-18 \mu \mathrm{m}$ (measured by using an optical microscope). An orifice is used at the second junction to better control and reduce droplet size (Um et al. 2008; Velasco et al. 2012) (Figure 2a). The total length of the main stream after the orifice is adjusted to carry more than 50 droplets, so as to minimize the fluctuations in the hydrodynamic resistance and flow rate caused by droplets entering and exiting the main stream (Glawdel and Ren 2012a).

A long serpentine channel is placed after the orifice to provide homogeneous mixing. 3D motion occurs in each half of the droplet due to symmetric vortices while droplets are travelling through straight channels. Mixing between the two halves of the droplet is dominated by molecular diffusion. To ensure homogeneous mixing across the entire droplet, the symmetry must be disturbed by pumping droplets through serpentine channels (Song et al. 2003b, 2006; Shestopalov et al. 2004). Non-axisymmetric vortexes inside droplets induced by the time periodic recirculating flow inside plug-shaped droplets results in chaotic advection. This recirculating flow is the result of shearing interaction between the channel wall and the fluid of the droplet. The chaotic flow happening inside the droplet can be interpreted by using the Baker's transformation (Song et al. 2003a). The thickness of layers of fluids is calculated by:

$$
\text { stl }(n)=\operatorname{stl}(0) \times 2^{-n}
$$

where $n$ is the number of chaotic cycles. The mixing time is estimated by:

$$
\mathrm{t}_{\mathrm{mix}, \mathrm{ca}} \sim\left(\frac{\mathrm{aw}}{\mathrm{U}}\right) \log (\mathrm{Pe})
$$

where $a$ is the dimensionless length of the plug measured relative to the channel width, $w$. $P e$ is the Peclet number. Based on the range of operating conditions, the serpentine channel is designed to have 20 turns with an inner and outer diameter of 150 and $200 \mu \mathrm{m}$, respectively.

\section{Experimental Validation}

\subsection{Materials and Experiment setup}

\subsubsection{Device Fabrication}

Microfluidic devices were fabricated using standard soft-lithography.(Xia and Whitesides 1998; Kim et al. 2008) Briefly, SU8 -2015 negative photoresist (MicroChem) was used to form the micro-mold containing the microchannels. For replica molding, PDMS (Sylgard 184 Dow Corning) base and curing agent were mixed and degassed at a ratio of 1:9, poured on top of the mold and baked at $95^{\circ} \mathrm{C}$ for 2 hours. The PDMS substrate was then peeled and cleaned with isopropyl alcohol before being bonded to a clean glass slide coated with a thin film of PDMS exposed to oxygen plasma treatment. Inlets and outlets were made using a $1.5 \mathrm{~mm}$ biopsy punch. To achieve wetting stability, the microfluidic chip was placed on a hot plate at $110^{\circ} \mathrm{C}$ for 2 days rendering its surface hydrophobic before the experiment.

\subsubsection{Materials}


Green-emitting CdSe/ZnS core/shell quantum dots (photoluminescence, PL at $518 \mathrm{~nm}$ ) were from Cytodiagnostics (Burlington, ON, Canada). Diethylaminoethyl (DEAE)-functionalized magnetic beads (MB, 1 um) were from Bioclone Inc. (San Diego, CA). The capturing buffer for immobilizing QDs on MBs was Tris-borate buffer (100 mM, $\mathrm{pH}$ 7.4) with $20 \mathrm{mM} \mathrm{NaCl}$. The buffer for releasing QDs from MBs was borate buffer (100 mM, pH 10) with $1 \mathrm{M}$ $\mathrm{NaCl}$. The $5 \mathrm{CSt}$ silicon oil was from Sigma Aldrich Canada. Water-soluble quantum dots were prepared from the oleic acid-capped $\mathrm{CdSe} / \mathrm{ZnS}$ quantum using a previously reported ligand exchange procedure with glutathione (GSH) (Noor et al. 2013a). Approximately $0.2 \mathrm{~g} \mathrm{GSH}$ was dissolved in $600 \mu \mathrm{L}$ of TMAH. Then 700 pmol of QDs was dissolved in $2 \mathrm{~mL}$ of chloroform that was gradually added to the GSH solution. Thereafter, the solution was briefly agitated and incubated overnight at room temperature. After incubation, the GSH-modified QDs (GSH-QDs) were extracted to a top layer of $200 \mu \mathrm{L}$ of borate buffer (BB) containing $250 \mathrm{mM} \mathrm{NaCl}$, at $\mathrm{pH}$ of 9.2 . The organic layer was discarded and the aqueous layer was transferred to a $1.5 \mathrm{~mL}$ centrifuge tube. Ethanol was added to the QD solution until the solution became turbid. The mixtures were centrifuged at $8000 \mathrm{rpm}$ for $7 \mathrm{~min}$ to obtain a pellet of QDs. The buffer addition, ethanol precipitation and centrifugation was repeated two more times. Finally, GSH-QDs were redispersed in a borate buffer at $\mathrm{pH} 9.2$ without $\mathrm{NaCl}$. The concentration of QDs was determined using UV-vis absorption spectroscopy. An extinction coefficient value of $284,000 \mathrm{~L} \mathrm{~mol}^{-1} \mathrm{~cm}^{-1}$ was used to complete the calculations based on the absorption of the QDs with the first excitation peak at $\sim 500 \mathrm{~nm}$.

\subsubsection{Experimental setup}

The experimental system consisted of two main parts: 1) a pressure system (MSFC 4C, Fluigent) that was used to pump the fluids through microchannels, and 2) an inverted microscope (Nikon Ti-Eclipse) that was used to record the dynamic flow of droplets and detect luminescence. The conjugation processes were visualized using the microscope with $40 \mathrm{x}(\mathrm{N} / \mathrm{A}: 0.75 \mathrm{~mm})$ and $20 \mathrm{x}(\mathrm{N} / \mathrm{A}: 0.45 \mathrm{~mm})$ objectives. The excitation sources were a $100 \mathrm{~W}$ LED lamp (Nikon) for bright field images and $100 \mathrm{~W}$ mercury halide lamp (Nikon) for fluorescence images. All images were captured using a CCD camera (Q-imaging R2000), or a high-speed CMOS camera (Phantom v210, Vision Research). A fluorescein isothiocyanate (FITC) filter cube was used within the microscope.

\subsection{Experimental Procedure}

\subsubsection{Co-encapsulation of $Q D$ s and a single $M B$}

The stock solution of QDs solution was diluted to $1 \mathrm{nM}$ (unless noted otherwise) using the capturing buffer (Trisborate, $100 \mathrm{mM}, \mathrm{pH}$ 7.4) for the final experiment. The saturated concentration is approximately 270 pmol QDs per 1 mg MB (Sedighi and Krull 2016). Following the step-by-step protocol provided by Bioclone Inc., $1 \mu \mathrm{l}$ of the MB solution supplied by the manufacturer was washed two times with the releasing borate buffer $(100 \mathrm{mM}, \mathrm{pH} 10)$, and then diluted by the capturing Tris-borate buffer $(100 \mathrm{mM}, \mathrm{pH}$ 7.4) mixed with $80 \%$ (wt \%) glycerol. The viscosity of the mixture of glycerol buffer was $\sim 44.1 \mathrm{mPa}$.s, while the viscosity of the mixture of quantum dots buffer was $\sim 1.02$ $\mathrm{mPa}$.s. The resulting concentration of MBs was $\sim 10^{7}$ beads $/ \mathrm{mL}$. The microfluidic chip was primed with silicon oil for 20 minutes for good wetting conditions and thus stable droplet formation. The encapsulation process was controlled by utilizing two side streams (dispersed phase 2) to squeeze the middle stream (dispersed phase 1), resulting in a focusing stream (Figure 3). The droplets were generated under the squeezing regime, which the Capillary is kept in the range from 0.002 to 0.006 , and the ratio between the dispersed phase and continuous phase is $\frac{1}{8} \leq \varphi=\frac{Q_{d}}{Q_{c}} \leq \frac{1}{4}$. In details, the flow rate of the continuous phase was set at the range from 0.4 to $0.8 \mathrm{ul} / \mathrm{min}$; whereas, the total flow rate of the dispersed phase was kept as constant $\sim 0.1 \mathrm{ul} / \mathrm{min}$. The single encapsulation is efficient when the ratio of dispersed phase 1 and dispersed phase $2,(\lambda)$, is from 0.6 to 0.8 . The range of $(\lambda)$ ratio used in our experiment is also similar to the one, which is reported by X. Chen et al. (Chen and Ren 2017). So that, the $1 \mu \mathrm{m}$ MBs were kept inside the inner stream since the highly viscous inner fluid acted as a soft wall, and encapsulation into a droplet with the QDs occurred at the second junction. In addition, by varying the droplet size, the results suggested that there was a 1 um MB per droplet, when $1.2 \leq L^{*} \leq 2.5$ and $L^{*}=L_{\text {droplet }} / W_{\text {channel }}$ is the dimensionless droplet length. 


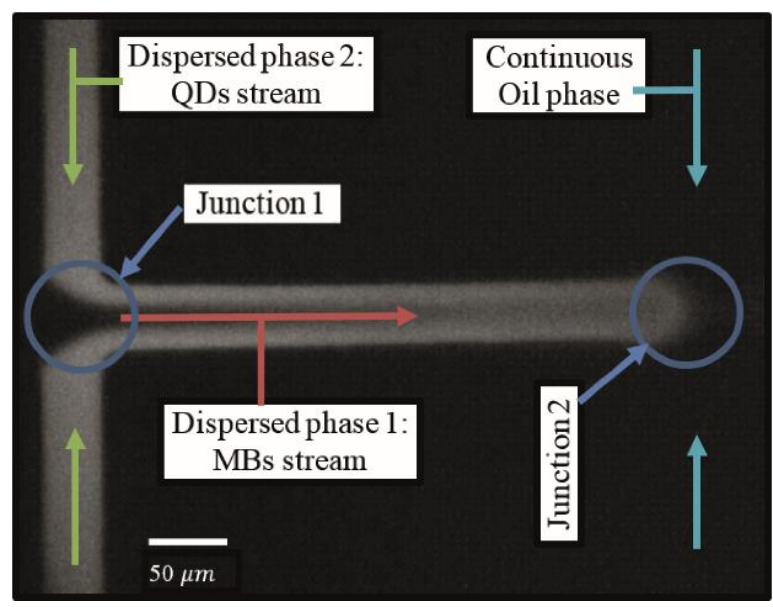

Fig 3. Using stratified flow with viscosity contrast in order to align a train of MBs and enhance co-encapsulation of a single MB and many QDs into a single droplet

Three bead sizes $-1 \mu \mathrm{m}, 4 \mu \mathrm{m}$, and $10 \mu \mathrm{m}$ - were used to test the effectiveness of the flow system. By counting the number of droplets and frames captured during a droplet generation cycle using the high-speed CMOS camera, the efficiency of encapsulation of a single $1 \mu \mathrm{m} \mathrm{MB}$ was determined to be up to $70 \%$. (See the supplementary video 1,2 , 3 ). It was noted that miscounting of the beads that were not at the focal plane was possible, but negligible because the channel height was $25 \mu \mathrm{m}$ before swelling, and measured as $\sim 16$ to $18 \mu \mathrm{m}$ after swelling (Roach et al. 2005; Glawdel and Ren 2012a, b). It was observed that majority of the beads were near the focal plane due to wall-induced lift forces. Image processing was necessary to clearly visualize the single encapsulation of $1 \mu \mathrm{m}$ MBs (Figure 4).

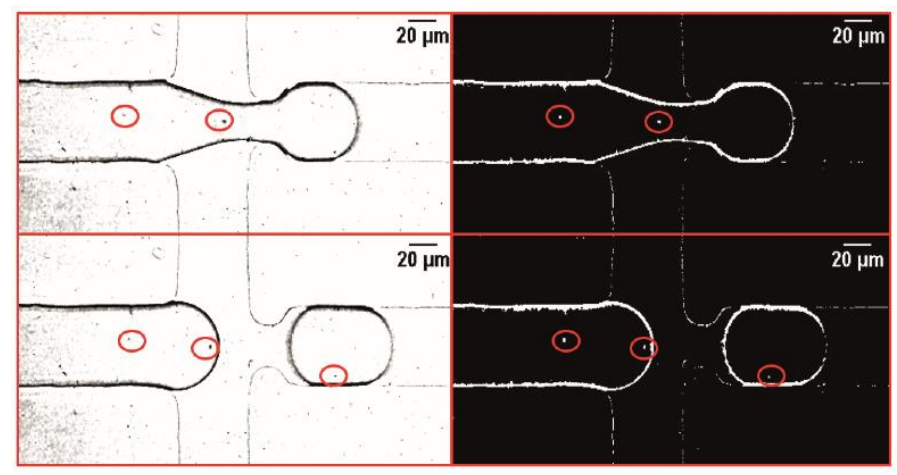

Fig 4. (Left) RGB images showing the single $1 \mu \mathrm{m}$ MB encapsulation. (Right) Binary images showing the single 1 um MB encapsulation.

\subsubsection{Conjugation process}

After a magnetic bead and a number of quantum dots are co-encapsulated into one single droplet, the electrostatic association occurred within 10 seconds and continued while the droplet was travelling through the serpentine channel (Figure 5). The serpentine channel consists of 20 turns which provides the 3D well-mixing inside droplets via chaotic convection. By comparing the intensity contrast between the empty droplets and the droplets that entrained a single $\mathrm{MB}$, it was demonstrated that this microfluidic device enabled rapid conjugation (supplemental material). 


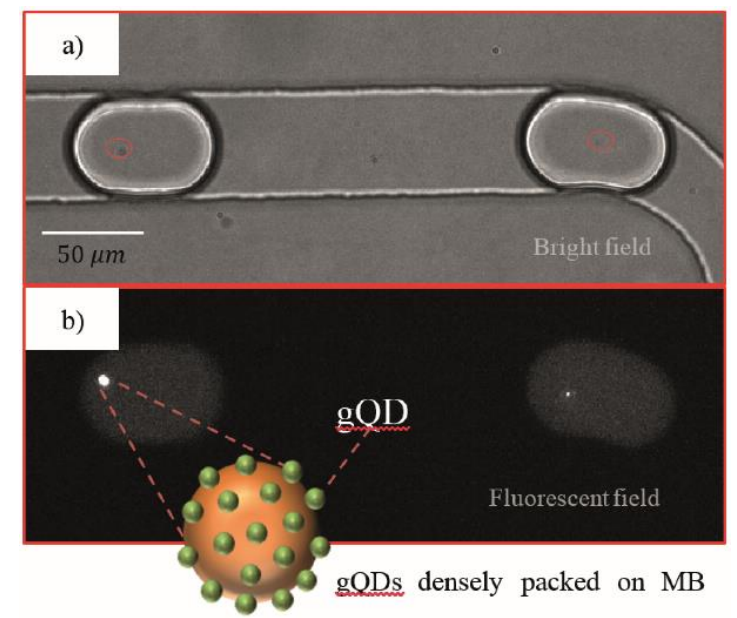

Fig 5. Zoom-in (40x magnification) images of fluorescence from QDs confirm the association with MBs in a $50 \mu \mathrm{m}$ sized channel. a) The image was taken under the bright field, resulting in gray scale image. b) The QD-MB conjugate is luminescent in the fluorescent field.

One of the disadvantages of batch processing is the use of large quantities of QDs to ensure a high-density coating of QDs on MBs. The consequence is a requirement for extensive washing to remove unbound QDs before proceeding to the subsequent step of QD conjugation to biomolecules. It was found that a microfluidic droplet used as a reaction compartment facilitated coating of MBs with a high density of QDs without using a large quantity of QDs. This is supported by two observations. First, the comparison of the fluorescent intensity between the inlet filled with the original solution containing QDs (before conjugation) and the outlet where there is a mixture of MB-QDs and excess QDs shows that the excess QDs after conjugation is negligible (supplemental material). Second, the fluorescent intensity of the recovered QDs which are detached from MBs using the releasing buffer is about $75 \%$ of the original QDs solution (Figure 6). There is some loss of QDs due to multiple pipetting steps required for preparation of the samples for the fluorescence spectroscopy measurements, suggesting that the actual extraction of QDs on MBs is even higher than $75 \%$. In contrast, batch processing would leave most of the QDs in solution. Therefore, the proposed droplet-based microfluidic platform has potential to facilitate subsequent bioconjugation of the MB-QDs (with molecules such as single stranded oligonucleotides), as it could be done by continuous flow without washing to remove excess QDs (Coopersmith et al. 2015), simplifying the process of nanoparticle decoration.

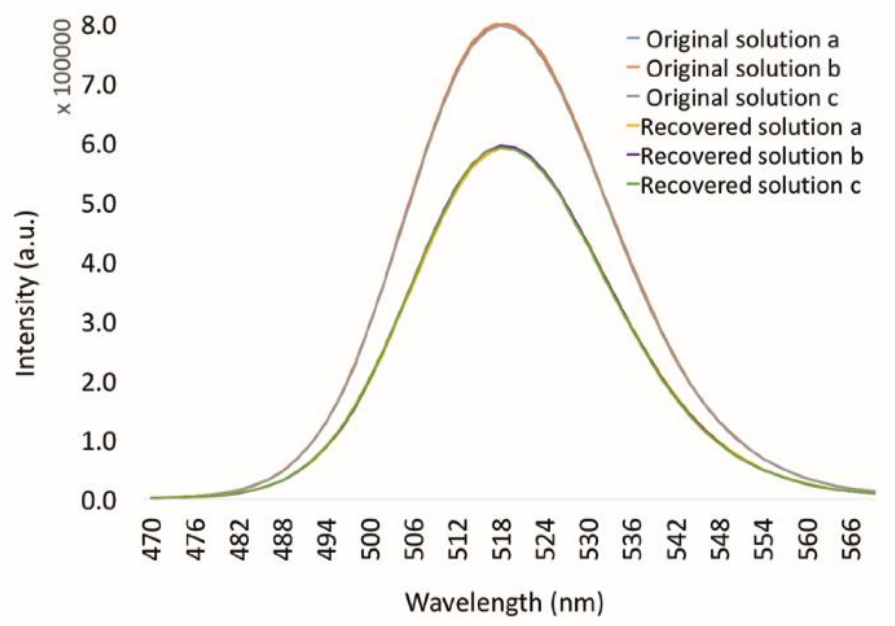

Fig 6. Comparison the intensity of original QDs solution before running experiment and the intensity of the recovery QDs. 
It is noteworthy that differences in the fluorescent intensity of the MB-QD conjugates between two adjacent droplets are primarily due to off-focus collection of signal. Improvement of the optical interrogation to control focal position makes possible quantitative evaluation of the extent of conjugation of QDs onto each single MB, offering a degree of quality control to adjust conjugation processes on-the-fly that is impossible for batch reactions. In order to confirm that the electrostatic adsorption onto single MBs does not alter the optical properties of QDs, the fluorescence spectra of the recovered and original QDs are compared. The emission profiles of the QDs before and after interaction with MBs are identical (Figure 6), confirming that the solid phase manipulation does not alter the spectral properties of the QDs. The indication that the QDs retain their spectral characteristics is essential for further applications, such as using QDs as donors in FRET-based bioassays.

\section{Conclusions}

Droplet microfluidic methods are currently used in various fields such as biotechnology, pharmaceuticals, and biochemistry for sample handling, synthetic reactions, and diagnostics. In this report, a droplet microfluidic platform is explored as a platform that could eventually support processes to decorate quantum dots with biomolecules. The droplet microfluidic approach has been demonstrated to be capable of encapsulating a single $1 \mu \mathrm{m}$ magnetic bead with many quantum dots in one aqueous droplet. Furthermore, a rapid electrostatic association of the QDs onto the MB surface has been demonstrated, with speed being associated with a chaotic advection inside nano-liter sized droplets. The efficiency of sample consumption was improved, and the aqueous droplets were extraordinarily uniform. The precision of droplet formation enables quantitative control of the reaction environment. The methodology is applicable to other types of nanoparticle (e.g. gold nanoparticles, up-conversion nanoparticles). The next step to further develop the droplet microfluidic reactor will involve the decoration QDs with biomolecules in continuous flow production, leading to a manufacturing platform.

\section{ASSOCIATED CONTENT}

\section{Supporting Information}

The following files are available free of charge.

Supplemental Information (PDF)

\section{Acknowledgements}

The authors acknowledge the financial support to Drs. U Krull and C Ren through a grant from the Natural Sciences and Engineering Research Council of Canada. Dr. C Ren also acknowledges support from the Canada Research Chair program, and the Canada Foundation for Innovation for facility support.

\section{References}

Algar WR, Krull UJ (2007) Towards multi-colour strategies for the detection of oligonucleotide hybridization using quantum dots as energy donors in fluorescence resonance energy transfer (FRET). Anal Chim Acta 581:193201. doi: 10.1016/j.aca.2006.08.026

Algar WR, Tavares AJ, Krull UJ (2010) Beyond labels: A review of the application of quantum dots as integrated components of assays, bioprobes, and biosensors utilizing optical transduction. Anal Chim Acta 673:1-25. doi: 10.1016/j.aca.2010.05.026

Boeneman K, Deschamps JR, Buckhout-white S, et al (2010) Quantum Dot DNA Bioconjugates : Architecture. ACS Nano 4:7253-7266

Bruchez Jr. M, Moronne M, Gin P, et al (1998) Semiconductor Nanocrystals as Fluorescent Biological Labels. Science (80- ) 281:2013-2016. doi: 10.1126/science.281.5385.2013 
Cao Y, Stavrinadis A, Lasanta T, et al (2016) The role of surface passivation for efficient and photostable PbS quantum dot solar cells. Nat Energy 1:16035. doi: 10.1038/nenergy.2016.35

Chen X, Glawdel T, Cui N, Ren CL (2015) Model of droplet generation in flow focusing generators operating in the squeezing regime. Microfluid Nanofluidics 18:1341-1353. doi: 10.1007/s10404-014-1533-5

Chen X, Ren CL (2017) Experimental study on droplet generation in flow focusing devices considering a stratified flow with viscosity contrast. Chem Eng Sci 163:1-10. doi: 10.1016/j.ces.2017.01.029

Chong ZZ, Tan SH, Ganan-Calvo AM, et al (2016) Active droplet generation in microfluidics. Lab Chip 16:35-58. doi: 10.1039/C5LC01012H

Chou LYT, Zagorovsky K, Chan WCW (2014) DNA assembly of nanoparticle superstructures for controlled biological delivery and elimination. Nat Nanotechnol 9:148-155. doi: 10.1038/nnano.2013.309

Coopersmith K, Han H, Maye MM (2015) Stepwise Assembly and Characterization of DNA Linked Two-Color Quantum Dot Clusters. Langmuir 31:7463-7471. doi: 10.1021/acs.langmuir.5b01130

Cutler JI, Auyeung E, Mirkin CA (2012) Spherical nucleic acids. J Am Chem Soc 134:1376-1391. doi: $10.1021 / \mathrm{ja} 209351 \mathrm{u}$

Garstecki P, Fuerstman MJ, Stone H a, Whitesides GM (2006) Formation of droplets and bubbles in a microfluidic Tjunction-scaling and mechanism of break-up. Lab Chip 6:437-446. doi: 10.1039/b510841a

Giri S, Sykes EA, Jennings TL, Chan WCW (2011) Rapid screening of genetic biomarkers of infectious agents using quantum dot barcodes. ACS Nano 5:1580-1587. doi: 10.1021/nn102873w

Glawdel T, Ren CL (2012a) Global network design for robust operation of microfluidic droplet generators with pressure-driven flow. Microfluid Nanofluidics 13:469-480. doi: 10.1007/s10404-012-0982-y

Glawdel T, Ren CL (2012b) Droplet formation in microfluidic T-junction generators operating in the transitional regime. III. Dynamic surfactant effects. Phys Rev E - Stat Nonlinear, Soft Matter Phys 86:1-9. doi: 10.1103/PhysRevE.86.026308

Günther A, Jensen KF (2006) Multiphase microfluidics: from flow characteristics to chemical and materials synthesis. Lab Chip 6:1487-1503. doi: 10.1039/B609851G

Han Y, Noor MO, Sedighi A, et al (2017) Inorganic Nanoparticles as Donors in Resonance Energy Transfer for SolidPhase Bioassays and Biosensors. Langmuir acs.langmuir.7b01483. doi: 10.1021/acs.langmuir.7b01483

He X, Li Z, Chen M, Ma N (2014) DNA-programmed dynamic assembly of quantum dots for molecular computation. Angew Chemie - Int Ed 53:14447-14450. doi: 10.1002/anie.201408479

Huo F, Lytton-Jean AKR, Mirkin CA (2006) Asymmetric functionalization of nanoparticles based on thermally addressable DNA interconnects. Adv Mater 18:2304-2306. doi: 10.1002/adma.200601178

Inoue A, Sugimoto H, Yaku H, Fujii M (2016) DNA assembly of silicon quantum dots/gold nanoparticle nanocomposites. RSC Adv 6:63933-63939. doi: 10.1039/C6RA13565J

Kaminski TS, Scheler O, Garstecki P (2016) Droplet microfluidics for microbiology: techniques, applications and challenges. Lab Chip 16:. doi: 10.1039/C6LC00367B

Kim J, Junkin M, Kim DH, et al (2009) Applications, techniques, and microfluidic interfacing for nanoscale biosensing. Microfluid Nanofluidics 7:149-167. doi: 10.1007/s10404-009-0431-8

Kim P, Kwon KW, Park MC, et al (2008) Soft Lithography for Microfluidics : a Review. Biochip J 2:1-11

Lalander CH, Zheng Y, Dhuey S, et al (2010) DNA-directed self-assembly of gold nanoparticles onto nanopatterned surfaces: Controlled placement of individual nanoparticles into regular arrays. ACS Nano 4:6153-6161. doi: $10.1021 / \mathrm{nn} 101431 \mathrm{k}$

Liu H, Shu W, Liu Z, et al (2017) A simple method of constructing microfluidic solid-state quantum dot molecular beacon array for label-free DNA detection. Microfluid Nanofluidics 21:1-9. doi: 10.1007/s10404-017-1906-7

Mattoussi H, Mauro JM, Goldman ER, et al (2000) Self-Assembly of CdS-ZnS Quantum Dot Bioconjugates Using an Engineered Recombinant Protein. J Am Chem Soc 122:12142-12150. doi: 10.1021/ja002535y

Noor MO, Shahmuradyan A, Krull UJ (2013a) Paper-based solid-phase nucleic acid hybridization assay using immobilized quantum dots as donors in fluorescence resonance energy transfer. Anal Chem 85:1860-1867. doi: $10.1021 / \mathrm{ac} 3032383$

Noor MO, Tavares AJ, Krull UJ (2013b) On-chip multiplexed solid-phase nucleic acid hybridization assay using spatial profiles of immobilized quantum dots and fluorescence resonance energy transfer. Anal Chim Acta 788:148-157. doi: 10.1016/j.aca.2013.06.017

Oh KW, Lee K, Ahn B, Furlani EP (2012) Design of pressure-driven microfluidic networks using electric circuit analogy. Lab Chip 12:515-45. doi: 10.1039/c2lc20799k

Peng H, Zhang L, Kjällman THM, et al (2007) DNA hybridization detection with blue luminescent quantum dots and dye-labeled single-stranded DNA. J Am Chem Soc 129:3048-3049. doi: 10.1021/ja0685452

Petryayeva E, Algar WR, Medintz IL (2013) Quantum dots in bioanalysis: A review of applications across various 
platforms for fluorescence spectroscopy and imaging. Appl Spectrosc 67:215-252. doi: 10.1366/12-06948

Prabhu VM, Hudson SD (2009) Nanoparticle assembly: DNA provides control. Nat Mater 8:365-366. doi: $10.1038 /$ nmat 2436

Resch-Genger U, Grabolle M, Cavaliere-Jaricot S, et al (2008) Quantum dots versus organic dyes as fluorescent labels. Nat Methods 5:763-775. doi: 10.1038/nmeth.1248

Roach LS, Song H, Ismagilov RF (2005) Controlling nonspecific protein adsorption in a plug-based microfluidic system by controlling interfacial chemistry using fluorous-phase surfactants. Anal Chem 77:785-796. doi: $10.1021 / \mathrm{ac} 049061 \mathrm{w}$

Rosenfeld L, Lin T, Derda R, Tang SKY (2014) Review and analysis of performance metrics of droplet microfluidics systems. Microfluid Nanofluidics 16:921-939. doi: 10.1007/s10404-013-1310-x

Sedighi A, Krull UJ (2016) Rapid Immobilization of Oligonucleotides at High Density on Semiconductor Quantum Dots and Gold Nanoparticles. Langmuir 32:13500-13509. doi: 10.1021/acs.langmuir.6b03840

Shestopalov I, Tice JD, Ismagilov RF (2004) Multi-step synthesis of nanoparticles performed on millisecond time scale in a microfluidic droplet-based system. Lab Chip 4:316-321. doi: 10.1039/b403378g

Song H, Bringer MR, Tice JD, et al (2003a) Experimental test of Scaling of mixing by chaotic advection in droplets moving through microfluidic channels. Appl Phys Lett 83:4664-4666. doi: 10.1097/OPX.0b013e3182540562.The

Song H, Chen DL, Ismagilov RF (2006) Reactions in Droplets in Microfluidic Channels. Angew Chem Int Ed Engl 45:7336-7356. doi: 10.1097/OPX.0b013e3182540562.The

Song H, Tice JD, Ismagilov RF (2003b) A Microfluidic System for Controlling Reaction Networks in Time - Song 2003 - Angewandte Chemie - Wiley Online Library. Angew Chemie 115:792-796. doi: 10.1002/ange.19350483406

Stanisavljevic M, Krizkova S, Vaculovicova M, et al (2015) Quantum dots-fluorescence resonance energy transferbased nanosensors and their application. Biosens Bioelectron 74:562-574. doi: 10.1016/j.bios.2015.06.076

Sun D, Gang O (2013) DNA-Functionalized Quantum Dots : Fabrication, Structural , and Physicochemical Properties. doi: 10.1021/la4000186

Suzuki M, Husimi Y, Komatsu H, et al (2008) Quantum Dot FRET Biosensors that Respond to pH, to Proteolytic or Nucleolytic Cleavage, to DNA Synthesis, or to a Multiplexing Combination. J Am Chem Soc 130:5720-5725. doi: $10.1021 / \mathrm{ja} 710870 \mathrm{e}$

Tan BW, Takeuchi S (2007) Monodisperse Alginate Hydrogel Microbeads for Cell Encapsulation **. Adv Mater 19:2696-2701. doi: 10.1002/adma.200700433

Teh S-Y, Lin R, Hung L-H, Lee AP (2008) Droplet microfluidics. Lab Chip 8:198-220. doi: 10.1039/b715524g

Um E, Lee D-S, Pyo H-B, Park J-K (2008) Continuous generation of hydrogel beads and encapsulation of biological materials using a microfluidic droplet-merging channel. Microfluid Nanofluidics 5:541-549. doi: 10.1007/s10404-008-0268-6

Velasco D, Tumarkin E, Kumacheva E (2012) Microfl uidic Encapsulation of Cells in Polymer Microgels. 16331642. doi: $10.1002 / \mathrm{smll} .201102464$

Xia Y, Whitesides GM (1998) SOFT LITHOGRAPHY. Annu Rev Mater Sci 28:153-184

Xing Y, Rao J (2008) Quantum dot bioconjugates for in vitro diagnostics \& in vivo imaging. Cancer Biomark 4:307319

Zhang C-Y, Yeh H-C, Kuroki MT, Wang T-H (2005) Single-quantum-dot-based DNA nanosensor. Nat Mater 4:826831. doi: $10.1038 /$ nmat 1508

Zhu P, Wang L (2017) Passive and active droplet generation with microfluidics: a review. 17:34-75. doi: 10.1039/C6LC01018K

Zhu Y, Fang Q (2013) Analytica Chimica Acta Analytical detection techniques for droplet microfluidics - A review. Anal Chim Acta 787:24-35. doi: 10.1016/j.aca.2013.04.064 